\title{
Time-Frequency Analysis of EEG Spectrograms using 2-D Gabor Filters for Epileptic Seizure Classification
}

\author{
Ricardo Ramos-Aguilar ${ }^{1}$, J. Arturo Olvera-López ${ }^{1}$, Ivan Olmos-Pineda ${ }^{1}$, \\ Manuel Martín-Ortíz ${ }^{2}$ \\ 1 Autonomous University of Puebla, Faculty of Computer Science, Puebla, Mexico \\ ricramosa1@gmail.com, \{aolvera,iolmos\}@cs.buap.mx \\ 2 Information Tecnology and Communications Department (DCyTIC), National \\ Laboratory of Supercomputing (LNS - BUAP - CONACYT), Puebla, Mexico \\ manuel.martin@correo.buap.mx
}

\begin{abstract}
EEG analysis of epileptic seizure is a nonstationary and changing process; these EEGs contain multiple frequencies and use only conventional methods based on frequency or time which limit their analysis. In this paper, texture representation of Gabor filter response based on a spectrogram applying a STFT is proposed to classify epileptic and healthy states. As initial part of our research, energy and entropy were extracted from the Gabor filters response; these features and statistical values were employed to train Support Vector Machines and multilayer Perceptron classifiers.
\end{abstract}

Keywords: EEG signals, Short time Fourier transform, Spectrogram, Gabor filter, Seizure classification.

\section{Introduction}

Epilepsy is a neurological disease or disorder distinguished by recurrent unprovoked seizures and caused by the transient and unexpected disturbance, occurring throughout the brain due to hypersynchronous neuronal discharges, which decrease and increase amplitudes of brain activity [7,21]. Epilepsy affects about $1-2 \%$ of the world population and it is divided into two types: focal epilepsy, which involves a part of the cerebral hemisphere and provokes symptoms in some parts of body or related with mental functions; the other kind of epilepsy is the generalized one, which involve the entire brain and produces bilateral motor symptoms, normally resulting in unconsciousness. For this latter kind of epilepsy, there is no specific age at which seizures occur[3].

Electroencephalography (EEG) is a brain monitoring method based on measurements of potentials generated by electrical activity. This test provides evidence of how the brain works over time, which is related to bodily functions such as the pumping of the heart, gland secretion, breathing, internal temperature, etc. EEGs are commonly used by scientists and physicians for analyzing 
brain functions and diagnosing neurological disorders, such as brain tumors, head injuries, sleep disorders, dementia, epilepsy, Alzheimer's, seizure disorder, attention deficit disorder, anxiety disorder, fetal alcohol syndrome, and autism, as well as monitoring the effects of anesthesia during surgery $[22,12,15,9,8]$.

Nowadays, EEG is more used to diagnose and treat neuro-degenerative diseases and abnormalities. Usually, an EEG helps physicians to determine an accurate diagnosis; in neurology, a common application of the EEG is epilepsy detection, since it is a useful and inexpensive tool for showing the underlying manifestations of epilepsy[23]. In people who suffer from epilepsy, their EEG signal shows two categories of abnormal activity: ictal (during an epileptic seizure) and interictal (between seizures)[23,15]. EEG Analysis for diagnosing epilepsy started in 1970, and since then it has been a changing problem because of EGG's non-stationary features; at present, most problems in seizure detection are related to find events (ictal and interictal) during epileptic seizures [15].

The detection of epileptic seizures in EEG signals has been analyzed using several methods, such as frequency and time-based methods, wavelet transforms, and Gabor filters $[21,15,14]$. However, the nature of the EEG signals is non-stationary and by implementing frequency or time techniques for their analysis[15] presents problems, as the features obtained from these methods do not provide enough information from EEG signals[2]. Time-frequency analysis, however, is a powerful tool that decomposes the signal into both time and frequency, making it possible to analyze non-stationary signals such as EEGs. Using these methods for EEG signals, they can be treated as images and divided in order to extract features from subimages. These techniques have shown good results in terms of accuracy for different applications[20]. Specifically, Short Time Fourier Transform (STFT) is an alternative that has previously used in epileptic seizure analysis by [25] and other recent works with competitive results in classification, where statistical, energy, and other features are extracted from spectrograms [7, $21,15,14,2]$.

In this initial research work, feature extraction based on a STFT of EEG signals including epileptic seizure activity is presented. This approach extracts features from 2-D Gabor filters images obtained from a spectrogram EEG signal. The feature vector is used as input for training an Artificial Neural Networks (ANN) and Support Vector Machine (SVM). According to our experiments, the proposed method produces acceptable accuracy results. The rest of the paper is organized as follows: Section 2, introduces the methodology and the related theories. Section 3 provides a description of EEG dataset and the experimental analysis. Section 4 presents our conclusions and future work.

\section{Methodology}

This work presents a methodology for the classification of EEG signals as healthy and as undergoing epileptic seizures, based on first order descriptors obtained from 2D Gabor filters applied to EEG signal spectrograms. Fig. 1 shows the methodology: STFT is first applied to EEG signals to obtain time-frequency 
mappings as images, which are then converted into 8-bit gray scale images and divided into five sub-bands corresponding to physiological rhythms on the EEG. 2D Gabor filters are used to extract texture descriptors, which are employed to train SVM and ANN classifiers and to classify EEG signals, showing the results in terms of accuracy.

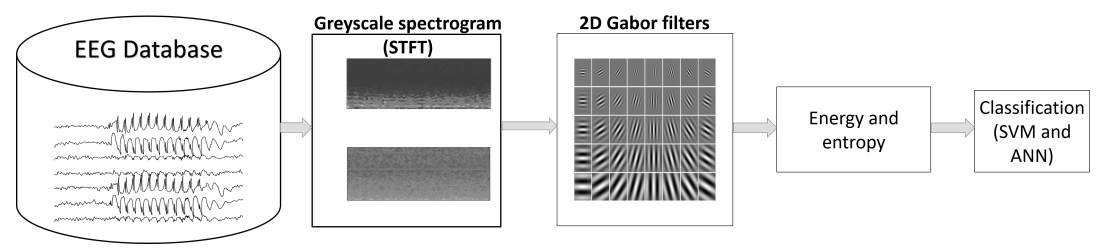

Fig. 1. Proposed methodology: From EEG Databases, spectrograms are computed and Gabor filters are applied to obtain features from the filters response.

\subsection{Short Time Fourier Transform}

A technique commonly used to analyze electrocardiogram (ECG) and EEG signals is STFT, which maps the signal into a two-dimensional function of frequency and time [18]. To retrieve information from the EEG, STFT is applied to the signal. STFT is employed to partition the EEG signal into several segments of short-time signals by shifting the time window with some overlapping [27], a process called windowing. Depending on the time windowing function $w[n]$, a spectrogram is classified as a narrowband or a wideband. When the time window is short, the STFT will be a wideband and a longer time returns a narrowband spectrogram[5]. The STFT general equation for a signal S is given by Equation (1):

$$
S(m, k)=\sum_{n=0}^{N-1} s(m-n) w(m) e^{-j \frac{2 \pi}{N} n k}
$$

Where

$k=[0: K]$ is the $K^{\text {th }}$ Fourier coefficient.

$K=N / 2$ is the frequency index corresponding to the Nyquist frequency.

$S(m, k)$ indicates the m-index time-frequency(frame) spectrogram.

$N=$ window segment length.

$N^{\prime}=$ the shifting step of the time window.

$w(n)=$ windowing method of an $n$ point sequence.

$N^{\prime}$ should be smaller than $N$ in order to produce an overlap between the time windows.

$S$ depends on the window function; in practice, different window shapes are used, such as: Symmetric, Unimodal and Gaussian. 
A spectrogram is a plot or visual mapping of the spectrum of frequencies of a signal along the time. This plot has a relation between time resolution and frequency resolution: a large window provides less localization in time and more discrimination in frequency. The window obtains a time-slice of the signal, during which the spectral characteristics are nearly constant [27], and the obtained segments shift the time window with some overlapping. The spectrogram is defined as the magnitude of $S(m, k)$, which is represented as $A(m, k)$ and computed as in equation (2):

$$
A(m, k)=\frac{1}{N}|S(m, k)|^{2} .
$$

Spectrogram resolution in time and frequency can change, depending on windowing; a wide window gives better frequency resolution but poor time resolution, while a narrow window produces better time resolution but poor frequency resolution. A visualization with different quality in the spectrogram depends on selecting an appropriate window length and overlapping. Fig. 2 shows the spectrogram of a signal, which is a time-varying spectral representation. The spectrogram layout is usually as follows: the $\mathrm{x}$-axis represents time, the $\mathrm{y}$-axis represents frequency, and the third dimension is amplitude (spectral content) of a frequency-time pair, which is color coded. It is a pseudo-3D plot where the intensity is represented by the z-axis.
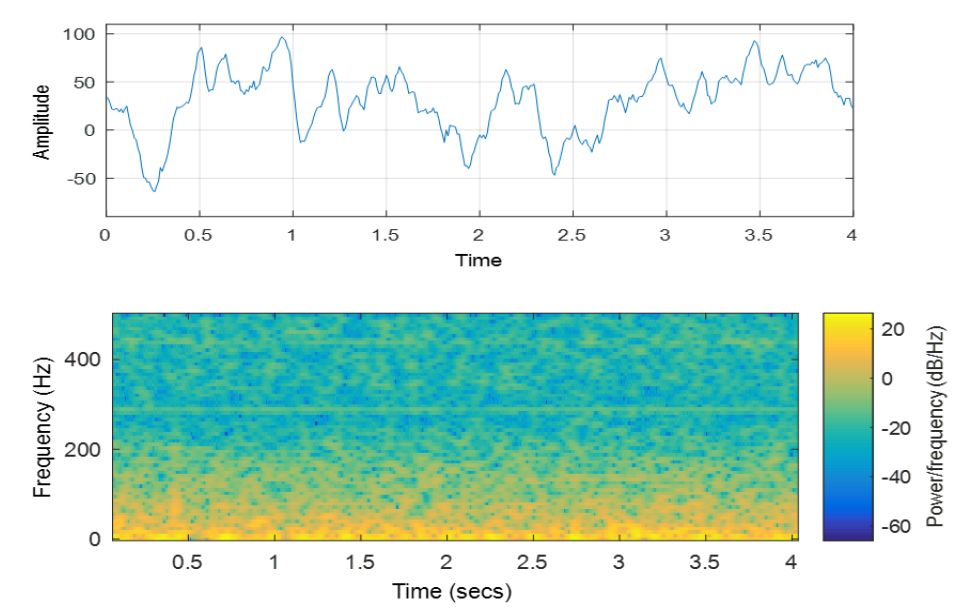

Fig. 2. EEG signal with different amplitude along time and its spectrogram.

\subsection{2-D Gabor Filters}

Gabor filtering is an option introduced by Daugman for analyzing textures in an image. Gabor filters model the response of simple cortical cells in the visual system and to extract image contours and directional textures [26]; these filters 
show an important advantage in their invariance to rotation, scale, translation, photometric disturbances, illumination, and image noise[10]. A 2D Gabor filter is a Gaussian kernel function modulated by a complex sinusoidal plane wave[26], and can be defined as:

$$
G(x, y)=\frac{f^{2}}{\pi \gamma} \exp \left(-\frac{x^{\prime 2}+\gamma^{2} y^{\prime 2}}{2 \sigma^{2}}\right) \exp \left(j 2 \pi f x^{\prime}+\phi\right),
$$

Where:

$$
\begin{gathered}
x^{\prime}=x \cos \theta+y \sin \theta, \\
y^{\prime}=-x \sin \theta+y \cos \theta .
\end{gathered}
$$

$f$ is the frequency of the sinusoid.

$\theta$ represents the orientation of the normal stripes in relation to the parallel stripes of the Gabor function.

$\phi$ is the phase offset.

$\sigma$ is the standard deviation of the Gaussian envelope.

$\gamma$ is the spatial aspect ratio that specifies the ellipticity of the support of Gabor function.

\subsection{Feature Representation}

The Gabor filter response from a spectrogram image is the convolution of Gabor filter with an EEG spectrogram $A(x, y)$. Hence, the response of Gabor filter is given as:

$$
R(x, y)=A(x, y) \circledast G(x, y) .
$$

Where $\circledast$ is the convolution operator.

Using a bank of filters with different parameters, it is possible to obtain features from spectrogram images with different scales and orientations and use them to retrieve local and discriminatory features[6]. A common texture feature is entropy, which is a measure of the amount of information (uncertainty) in a source; in the image field, it measures the randomness of the distribution of the coefficient values over the intensity levels[17]. In this case, the source is an image (spectrogram), which can be seen as a $2 \mathrm{D}$ information array[26]. The Shannon entropy is given by:

$$
H(X)=-\sum_{i=1}^{n} p\left(x_{i}\right) \log _{2} p\left(x_{i}\right) .
$$

In the previous equation, $P_{r}\left[X=x_{i}\right]=p\left(x_{i}\right)$ is the probability mass distribution of the source and $n$ is the number of probabilities $p\left(x_{i}\right)$.

Energy is another frequently used textural feature[16], which measures the uniformity of the intensity level distribution $[17,1]$ and proposes the energy in an 
analysis using Gabor filter texture. The energy is given by the following equation where $M$ and $N$ are the size of the sub-image a $(\mathrm{m}, \mathrm{n})$ :

$$
e(x)=\frac{1}{M N} \sum_{i=1}^{M} \sum_{i=1}^{N}|a(m, n)| .
$$

\subsection{Classification}

The energy and entropy features obtained are employed to train SVM and ANN classifiers, and distinguish between epileptic and healthy states. Support Vector Machine is implemented and its results are compared with Artificial Neural Network.

\subsection{Support Vector Machines}

An analysis technique in supervised learning is Support Vector Machine introduced by Vladimir Vapnik. The generated model outline the training data into different categories separated by a decision boundary. Trying to estimate a function $f: \mathbb{R}^{N} \rightarrow\{ \pm 1\}$ using training data, that is, $N$-dimensional patterns $x_{i}$ and class labels $y_{i}$, as follows:

$$
\left(x_{1}, y_{1}\right), \ldots,\left(x_{l}, y_{l}\right) \in \mathbb{R}^{N} \times\{ \pm 1\} .
$$

such that $f$ will correctly classify new examples $(\mathrm{x}, \mathrm{y})$ - that is, $f(x)=y$ for examples $(x, y)$, which were generated from the same underlying probability distribution $P(x, y)$ as the training data [11]. The decision boundary described by $(w, b)$, where $w$ is a vector containing the hyperplane parameters and $\mathrm{b}$ is an offset. The data is rescaled so that anything on or above the boundary $w^{T} x+b=1$ is of one class, and otherwise on or below the boundary is the other class. The in-between distance is called the margin defined as $\frac{2}{\|w\|^{2}}$. The maximal margin is equivalent to minimize $\|w\|$ which can be achieved through quadratic programming and the optimal hyperplane can be described by: $w=\sum^{\alpha_{i}} y_{i} x_{i}$. Nevertheless, separation task is not always linear due to data representation; hence, SVM transform the data from the input space into a higher dimensional space where is possible separate the data; projecting the data through kernel induced feature space, the kernel function deals with the dot product of the data. The optimal hyperplane can be expressed as equation (10) shows [24]:

$$
f(x)=\sum^{\alpha_{i}} y_{i} K\left(x_{i}, x\right)+b
$$

\subsection{Artificial Neural Networks}

McCullock and Pitts proposed a binary threshold unit as a computational model for an artificial method, which computes a weighted sum of $\mathrm{n}$ inputs signals, $x_{j},=$ 
Time-Frequency Analysis of EEG Spectrograms using 2-D Gabor Filters for Epileptic Seizure...

$1,2, \ldots, n$, and the output is 1 if the sum is above a specific thereshold $u$. Otherwise, the output is 0 . It is defined as:

$$
y=\Theta\left(\sum_{j=1}^{n} w_{j} x_{j}-u\right) .
$$

where $\theta($.$) is a unit step a function at 0$, and $w_{j}$ is the synapse weight associated with the $j^{\text {th }}$ input [13]. A classification problem can be defined by the function to use; some functions include step function, linear function and nonlinear sigmoid function. An Artificial Neural Network is constructed combining neurons, which are capable of solving complex real-life problems [24].

In supervised learning, the network is given a desired output for each pattern. Throughout learning, the actual output $y$ generated by the network may not equal the desired output $d$. A principle of error-correction learning rules is to use the error signal $(d-y)$ to update the connection weights and reduce the error; the perceptron learning rule is based on this error-correction principle. A common network is multilayer perceptron in which each computational unit employs either the thresholding function. This can be form arbitrarily complex decision boundaries and represent any function. Developing a back-propagation learning for determining weights in a multilayer perceptron has made these networks the most populars[13].

\section{Experimental Work}

\subsection{Epilepsy EEG Dataset}

The proposed methodology was tested using an open access Epilepsy EEG dataset from the Bonn University [4] with five subsets(Z,O,N,F and S), with each subset containing 100 EEG signals with 4097 samples, recorded at a sampling rate of $173.61 \mathrm{~Hz}$ using a 128-channel amplifier system with an average common reference. Sets Z and O were collected from five healthy volunteers. Sets N, F, and $\mathrm{S}$ were recorded from five epileptic patients for each set. Records from Set $\mathrm{S}$ were collected during seizure activity, while Sets $\mathrm{N}$ and $\mathrm{F}$ were gathered during seizure-free intervals. Fig. 3 summarizes the Bonn dataset and Fig. 4 shows EEG records from $\mathrm{S}$ and $\mathrm{Z}$ Sets, which were considered for our experimentation.

\subsection{Feature Extraction}

The proposed methodology is focused on extracting features from EEG spectrograms through 2D Gabor filters. As noted above, two sets are considered set S (people suffering an epileptic attack) and Z (healthy people); extracted features trough Gabor filters are employed to train SVM and ANN classifiers and by means of the extracted patterns the features quality is quantified. To develop 


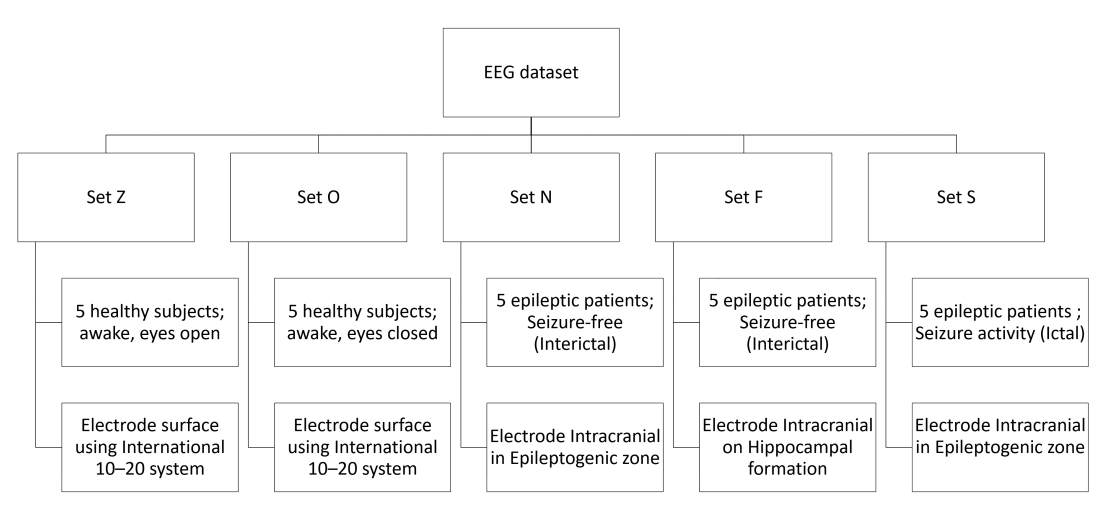

Fig. 3. A summary of Epilepsy EEG dataset.
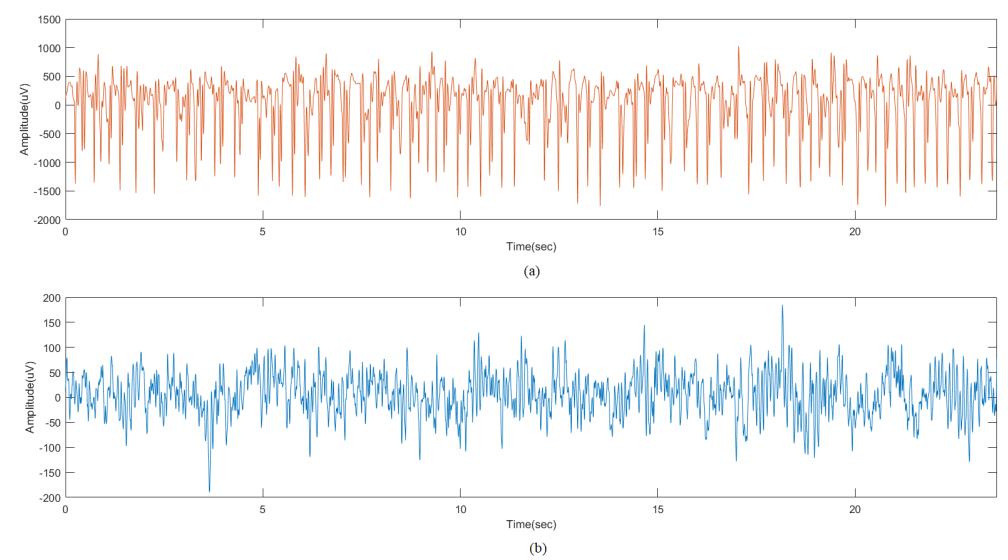

Fig. 4. An example of EEG signals. (a) a person suffering an epileptic attack and (b) a healthy person.

this proposal, Python 3.6 and the packages scikit-learn and scikit-image were used in our experimentation.

As Fig. 5 shows, STFT is first applied to EEG signals from both sets to obtain the spectrograms; the parameters used were the following: a Hanning window with 128 samples was used because of it showed the bests result versus other windows, the length of the Fast Fourier Transform nfft $=2000$, and the number of overlapped samples noverlap $=85$. The parameters were selected using 
[21] as a reference, because of the results obtained, however, that study did not implement a Hanning window.

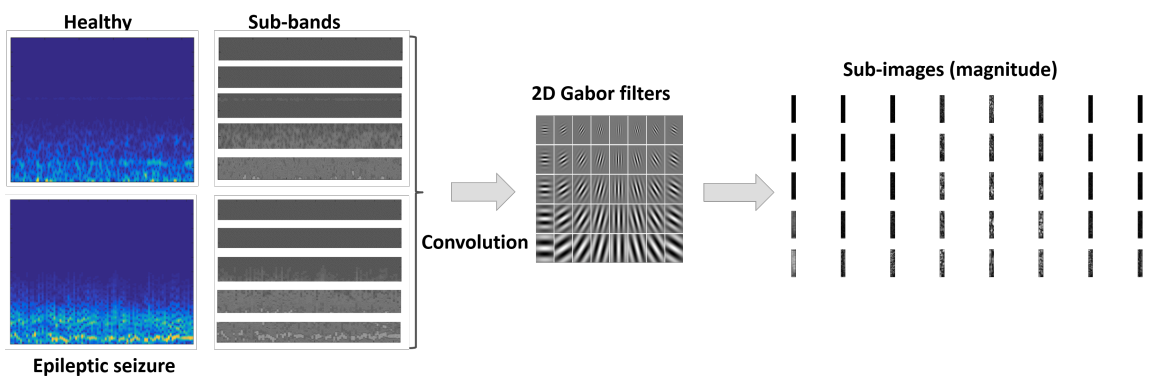

Fig. 5. Generation of the Gabor response from the spectrograms.

The obtained spectrograms were converted into 8-bit gray scale images and then divided into five sub-images related to frequency bands of the rhythms to localize significant structures. This was based on the bands proposed by [21]; other works, such as [7, 19], and [14], proposed dividing the spectrogram; however, they only use four bands, as the gamma band was omitted, since lost information was already taken into account. The ranges employed were: Delta $(0-4 \mathrm{~Hz})$, Theta $(4-8 \mathrm{~Hz})$, Alpha $(8-12 \mathrm{~Hz})$, Beta $(12-30 \mathrm{~Hz})$ and Gamma $(30-50 \mathrm{~Hz})$.

Gabor filters were applied to sub-images using five different frequencies and eight orientations; thus, the feature vector was $5 \times 8 \times(t f) \times 5 \times(s b)$; where $t f$ is textural features and $s b$ is sub-bands equal to 5 , as shown in Fig 5 . To extract the features, magnitudes responses of Gabor filters were considered.

\subsection{Experimental Results}

Two kinds of textural features (energy and entropy) were obtained and different vectors were generated by gathering the extracted features. To evaluate these features, SVM and with different kernels(linear, polynomial and RBF) and ANN, as different authors reported, such as [7,15,21], use this EEG dataset based on spectrograms. The classifiers performance was evaluated using accuracy as a metric, shown as percentage, which is given by the Equation (12); the presented values were obtained from an average of 10 experiments, as 10-fold cross validation was used, selecting the instances in a stratified way:

$$
\text { Accuracy }=\frac{T P+T N}{T P+F N+T N+F P} .
$$

Where:

$T P$-true positive is a seizure event detected by both the algorithm and the expert neurologist.

$T N$-true negative is a event identified as non-seizure by the algorithm and the 
expert neurologist.

$F P$-false positive is a seizure event detected by the algorithm, but not by the expert.

$F N$-false negative is a event identified as seizure by the expert, but not by the algorithm.

In the first experiment, energy was used as feature; hence, the feature vector has a dimension of 200 units. SVM and ANN were trained, obtaining different results, as shown in Table 1. The best accuracy was obtained through SVM-Polynomial, with 98\%. SVM-Linear and Perceptron had the same result, although the SVM standard deviation was lower, with 0.024 , meaning that 10 experiments of 10 folds were similar; using a perceptron classifier, the number of epochs was changed, using 100 and 500 and the result was the same.

Table 1. Energy feature classification results using SVM and Multilayer perceptron classifiers.

\begin{tabular}{|c|c|c|c|}
\hline Classifier & Parameters & Accuracy & rd Deviation \\
\hline$\overline{\overline{\text { SVM }}}$ & Polynomial & $98 \%$ & 0.024 \\
\hline$\overline{\mathrm{SVM}}$ & Linear & $97 \%$ & 0.033 \\
\hline$\overline{\mathrm{SVM}}$ & $\mathrm{RBF}$ & $96 \%$ & 0.044 \\
\hline Perceptron & $\begin{array}{l}\text { One hidden layer with } 10 \text { units, } \\
\text { backpropagation, and } 200 \text { epochs. }\end{array}$ & $95 \%$ & 0.042 \\
\hline Perceptron & $\begin{array}{l}\text { Three hidden layers with } 10,20 \\
2 \text { and } 10 \text { units, backpropagation, } \\
\text { and } 200 \text { epochs. }\end{array}$ & 96.5 & 0.032 \\
\hline Perceptron & $\begin{array}{l}\text { Two hidden layers with } 10 \text { and } \\
310 \text { units, backpropagation, and } \\
200 \text { epochs. }\end{array}$ & $97 \%$ & 0.024 \\
\hline
\end{tabular}

In addition, entropy was employed as a unique feature and the same classifiers were used as in the first experiment; SVM Polynomial shows the best accuracy, with $97.5 \%$, and the worst was SVM RBF, with 93\%; implementing a perceptron was obtained $94 \%$ as best accuracy. Nevertheless, energy has the best accuracy between both features. The results are shown in Table 2. Finally, entropy and energy were combined, obtaining a feature vector of 400 units, which were used to train SVM and ANN classifiers. The accuracy was better with a SVM Polynomial, resulting in an accuracy of $98.5 \%$ and the worst accuracy was 95.5\%, with SVM-RBF. Table 3 shows the results; although these are the best results with polynomial and linear SVM, employing only energy as a feature, the 10 experiments showed less change, with a standard deviation of 0.024 .

Some experiments suggest implementing mean and standard deviation of energy as textural features using Gabor filters [16,1]. However, using standard deviation and mean energy, the accuracy was less than $80 \%$ with SVM, while Perceptron with two hidden layers of 20 units reached 95\%. Employing stan- 
Time-Frequency Analysis of EEG Spectrograms using 2-D Gabor Filters for Epileptic Seizure...

Table 2. Entropy feature classification results using SVM and Multilayer perceptron classifiers.

\begin{tabular}{llcc}
\hline Classifier & Parameters & Accuracy & Standard Deviation \\
\hline \hline SVM & Polynomial & $97.5 \%$ & 0.04 \\
\hline SVM & Linear & $97 \%$ & 0.051 \\
\hline SVM & RBF & $93 \%$ & 0.051 \\
\hline Perceptron & $\begin{array}{l}\text { One hidden layer with 10 units, } \\
\text { backpropagation, and 200 epochs. }\end{array}$ & $92 \%$ & 0.090 \\
\hline $\begin{array}{l}\text { Two hidden layers with 10 and } \\
\text { Perceptron } 5\end{array}$ & $\begin{array}{l}10 \text { units, backpropagation, and } \\
\text { 200 epochs. }\end{array}$ & $93.5 \%$ & 0.084 \\
\hline $\begin{array}{l}\text { Three hidden layers with 10, 50 } \\
\text { Perceptron } 6 \\
\text { and 50 units, backpropagation, } \\
\text { and 200 epochs. }\end{array}$ & $94 \%$ & 0.070 \\
\hline
\end{tabular}

Table 3. Classification results with entropy and energy features using SVM and Multilayer perceptron classifiers.

\begin{tabular}{|c|c|c|c|}
\hline Classifier & Parameters & \multicolumn{2}{|c|}{ Accuracy Standard Deviation } \\
\hline$\overline{\overline{\text { SVM }}}$ & Polynomial & $98.5 \%$ & 0.032 \\
\hline$\overline{\mathrm{SVM}}$ & Linear & $98 \%$ & 0.04 \\
\hline SVM & $\mathrm{RBF}$ & $95.5 \%$ & 0.0437 \\
\hline Perceptron 7 & $\begin{array}{l}\text { One hidden layer with } 10 \text { units, } \\
\text { backpropagation, and } 200 \text { epochs. }\end{array}$ & $95.5 \%$ & 0.052 \\
\hline Perceptron & $\begin{array}{l}\text { Three hidden layers with } 10,40 \text { and } \\
50 \text { units,backpropagation, and } \\
200 \text { epochs. }\end{array}$ & $96.5 \%$ & 0.032 \\
\hline Perceptron 9 & $\begin{array}{l}\text { Two hidden layers with } 50 \text { and } \\
50 \text { units, backpropagation, and } \\
200 \text { epochs. }\end{array}$ & $97 \%$ & 0.04 \\
\hline
\end{tabular}

dard deviation and mean to entropy, however, SVM and Perceptron achieved less than $80 \%$. Finally, concatenating four features, the accuracy was $95 \%$ for SVM Polynomial, $92.5 \%$ for linear SVM, $82.5 \%$ for SVM RBF, and $94.5 \%$ for Perceptron. Some works as $[18,19,2,21]$ extracted different textural features, in this work one or two kind of features were employed and the results were similar to other approaches.

The obtained results are compared against reported results of other proposals which implement STFT and different features and epilepsy EEG dataset to the same sets S and Z, see table 4. In [7] and [14] five features were obtained with accuracies between $95.78 \%$ and $100 \%$, [15] extracted only energy as feature with results between $92.36 \%$ and $99.5 \%$. This proposal achieved $98.5 \%$ with two features energy and entropy, and this results are better than some experiments. 
Table 4. Comparison against other methods.

\begin{tabular}{lll}
\hline Reference & Method & Classification \\
\hline \hline Duque, et al,. 2014 & $\begin{array}{l}\text { Stochastic analysis and } \\
\text { cepstrals coefficients. }\end{array}$ & $95.78 \%-100 \%, \mathrm{SVM}$ \\
\hline Kovács, et al., 2014. & $\begin{array}{l}\text { MalmquistTakenaka } \\
\text { coefficients and statistical } \\
\text { features }\end{array}$ & $\begin{array}{l}96.7 \%, 98.36 \% \text {, and } 99.7 \% \\
\text { alternating decisiontree }\end{array}$ \\
\hline Kumar and Sharma,2015 & Energy and PCA. & ANN(99.5\%, 99.33\% and 92.36\%) \\
\hline Proposed method & $\begin{array}{l}\text { Gabor filters, energy } \\
\text { and entropy. }\end{array}$ & $\mathbf{9 8 . 5 \%} \mathbf{S V M}$ \\
\hline
\end{tabular}

\section{Conclusions}

In this paper, an alternative methodology for classifying epileptic seizures is proposed. STFT is used to represent EEG signals as spectrograms and analyze a signal as an image. 2D Gabor filters are employed to extract textural features and SVM and ANN are implemented to classify. Energy and entropy are used as features, however, probably, energy is the predominant feature due to in the most the classifiers, it was better than entropy and the best performance was obtained using SVM Polynomial for any feature. The mean and the standard deviation were not as relevant as in other works; however, with EEG spectrograms, these have been reported showing the behavior of the experiments.

Using the proposed methodology and changing different parameters, such as the length, type and overlapping of the STFT window, accuracy could improve.

From analyzed works, it could be noted that the spectrograms spectral peaks have been analyzed, hence, as future work, it is proposed to analyze the peaks employing clustering techniques to obtain features from the formed clusters and use a method to evaluate features and eliminate some of them non relevant.

Acknowledgments. The first author thanks the support from CONACyT-Mexico through the scholarship number 457637.

\section{References}

1. Al-Kadi, O.S.: A gabor filter texture analysis approach for histopathological brain tumor subtype discrimination. Journal of Science and Technology 12(22), 1-14 (2017)

2. Alçin, Ö.F., Siuly, S., Bajaj, V., Guo, Y., Şengür, A., Zhang, Y.: Multi-category eeg signal classification developing time-frequency texture features based fisher vector encoding method. Neurocomputing 218, 251 - 258 (2016)

3. Ambulkar, N.K., Sharma, S.N.: Detection of epileptic seizure in eeg signals using window width optimized s-transform and artificial neural networks. In: 2015 IEEE Bombay Section Symposium (IBSS). pp. 1-6 (2015)

4. Andrzejak, R.G., Lehnertz, K., Mormann, F., Rieke, C., David, P., Elger, C.E.: Indications of nonlinear deterministic and finite-dimensional structures in time 
series of brain electrical activity: Dependence on recording region and brain state. Phys. Rev. E 64, 1-8 (2001)

5. Boashash, B.: Heuristic formulation of time-frequency distributions. In: Boashash, B. (ed.) Time-Frequency Signal Analysis and Processing, pp. 65-102. Academic Press, Oxford, second edn. (2016)

6. Dora, L., Agrawal, S., Panda, R., Abraham, A.: An evolutionary single gabor kernel based filter approach to face recognition. Engineering Applications of Artificial Intelligence 62, $286-301$ (2017)

7. Duque-Muñoz, L., Espinosa-Oviedo, J.J., Castellanos-Dominguez, C.G.: Identification and monitoring of brain activity based on stochastic relevance analysis of short-time eeg rhythms. BioMedical Engineering OnLine 13(1), 1-20 (2014)

8. Gandhi, V.: Chapter 2 - interfacing brain and machine. In: Gandhi, V. (ed.) BrainComputer Interfacing for Assistive Robotics, pp. 7-63 (2015)

9. Grossi, E., Olivieri, C., Buscema, M.: Diagnosis of autism through eeg processed by advanced computational algorithms: A pilot study. Computer Methods and Programs in Biomedicine 142, 73-79 (2017)

10. Haghighat, M., Zonouz, S., Abdel-Mottaleb, M.: Cloudid: Trustworthy cloud-based and cross-enterprise biometric identification. Expert Systems with Applications 42(21), $7905-7916(2015)$

11. Hearst, M.A., Dumais, S.T., Osuna, E., Platt, J., Scholkopf, B.: Support vector machines. IEEE Intelligent Systems and their Applications 13(4), 18-28 (1998)

12. Ilyas, M.Z., Saad, P., Ahmad, M.I.: A survey of analysis and classification of eeg signals for brain-computer interfaces. In: 2015 2nd International Conference on Biomedical Engineering (ICoBE). pp. 1-6 (2015)

13. Jain, A.K., Mao, J., Mohiuddin, K.: Artificial neural networks: A tutorial. IEEE Computer 29, 31-44 (1996)

14. Kovacs, P., Samiee, K., Gabbouj, M.: On application of rational discrete short time fourier transform in epileptic seizure classification. In: 2014 IEEE International Conference on Acoustics, Speech and Signal Processing (ICASSP). pp. 5839-5843 (2014)

15. Kumar, J.S., Bhuvaneswari, P.: Analysis of electroencephalography (eeg) signals and its categorizationa study. Procedia Engineering 38, 2525-2536 (2012)

16. Li, W., Mao, K., Zhang, H., Chai, T.: Selection of gabor filters for improved texture feature extraction. In: 2010 IEEE International Conference on Image Processing. pp. 361-364 (2010)

17. Malik, F., Baharudin, B.: The statistical quantized histogram texture features analysis for image retrieval based on median and laplacian filters in the dct domain. Int. Arab J. Inf. Technol. 10, 616-624 (2013)

18. Mustafa, M., Taib, M.N., Murat, Z.H., Sulaiman, N., Aris, S.A.M.: The analysis of eeg spectrogram image for brainwave balancing application using ann. In: 2011 UkSim 13th International Conference on Computer Modelling and Simulation. pp. 64-68 (2011)

19. Mustafa, M., Taib, M.N., Murat, Z.H., Hamid, N.H.A.: Glcm texture classification for eeg spectrogram image. In: 2010 IEEE EMBS Conference on Biomedical Engineering and Sciences (IECBES). pp. 373-376 (2010)

20. Ridouh, A., Boutana, D., Bourennane, S.: Eeg signals classification based on time frequency analysis. Journal of Circuits, Systems and Computers 26(12), 1-26 (2017)

21. Şengür, A., Guo, Y., Akbulut, Y.: Time-frequency texture descriptors of eeg signals for efficient detection of epileptic seizure. Brain Informatics 3(2), 101-108 (2016) 
22. Siuly, S., Li, Y., Zhang, Y.: Electroencephalogram (EEG) and Its Background, pp. 3-21. Springer International Publishing, Cham (2016)

23. Siuly, S., Li, Y., Zhang, Y.: Significance of EEG Signals in Medical and Health Research, pp. 23-41. Springer International Publishing, Cham (2016)

24. Tawfik, N.S., Youssef, S.M., Kholief, M.: A hybrid automated detection of epileptic seizures in eeg records. Comput. Electr. Eng. 53(C), 177-190 (2016)

25. Tzallas, A. T., T.M.G., Fotiadis, D.I.: Epileptic seizure detection in eegs using time-frequency analysis. IEEE Trans. Inf. Technol. Biomed. 13, 703-710 (2009)

26. Vazquez-Fernandez, E., Dacal-Nieto, A., Martin, F., Torres-Guijarro, S.: Entropy of gabor filtering for image quality assessment. In: Campilho, A., Kamel, M. (eds.) Image Analysis and Recognition. pp. 52-61. Springer Berlin Heidelberg, Berlin, Heidelberg (2010)

27. Zabidi, A., Mansor, W., Lee, Y., Che Wan Fadzal, C.: Short-time fourier transform analysis of eeg signal generated during imagined writing. In: 2012 International Conference on System Engineering and Technology (ICSET). pp. 1-4 (2012) 